\title{
Tecnologías de la Información y las Comunicaciones: Telemedicina
}

\author{
Joseba Rabanales Sotos ${ }^{a}$, Ignacio Párraga Martíneza ${ }^{a}$, Jesús López-Torres Hidalgo ${ }^{a}$, \\ Fernando Andrés Pretel ${ }^{a}$, Beatriz navarro Bravo ${ }^{a}$
}

\begin{abstract}
a Unidad de Investigación de la Gerencia de Atención Primaria de Albacete.
\end{abstract}

Correspondencia: Joseba Rabanales Sotos, Unidad de Investigación, Gerencia de Atención Primaria de Albacete, C/ Dionisio Guardiola no 15$17,1^{\text {a }}$ Entreplanta, 02003 - Albacete, España. Telf.: 967195131, correo electrónico: rabanalessotos@hotmail.com

Recibido el 16 de septiembre de 2010 .

Aceptado para su publicación el 24 de noviembre de 2010.

\section{RESUMEN}

Las diferentes definiciones de telemedicina hacen referencia a las técnicas y tecnologías utilizadas para la práctica médica a distancia, manteniendo el significado etimológico del término telemedicina ("medicina a distancia"). La asistencia prestada a través de telemedicina facilita la accesibilidad a los servicios sanitarios, el seguimiento de las enfermedades crónicas y la educación sanitaria del paciente en medicina preventiva y salud publica. A pesar del aumento de la aplicación de las nuevas tecnologías al ámbito sanitario, la telemedicina no disfruta de una presencia mayoritaria en la actividad clínica y asistencial.

Palabras clave: Telemedicina, e-Salud, Tecnologías de la Información y la Comunicación.

\section{ABSTRACT}

Information Technology and Communications: Telemedicine

The different definitions of telemedicine refer to the techniques and technologies used for practicing medicine at a distance, thus maintaining the etymology of the term telemedicine ("medicine at a distance"). Telematic systems provide access to healthcare services, enable follow-up of chronic diseases and patient education in preventive medicine and public health. Despite the increase of applying these new technologies to the healthcare setting, telemedicine is still not widely used in clinical and healthcare activities.

Key words. Telemedicine, e-Health, Information and Communication Technologies.

\section{¿QUÉ ES LA TELEMEDICINA?}

Entre las diferentes definiciones utilizadas para el término telemedicina podemos adoptar, por su utilización más frecuente, aquella que la considera como una manera de proveer servicios sanitarios a pacientes en los que el acceso a los mismos está limitado por la geografía, el trabajo o la presencia de una enfermedad. En estos casos, la telemedicina puede mejorar la accesibilidad y la eficiencia de esos servicios ${ }^{1}$. En este mismo sentido, la OMS se refiere a la telemedicina como "el suministro de servicios de atención sanitaria en los casos en que la distancia es un factor crítico, llevado a cabo por profesionales sanitarios que utilizan tecnologías de la información y la comunicación para el intercambio de información válida para hacer diagnósticos, prevención y tratamiento de enfermedades, formación continuada de profesionales en atención a la salud, así como para actividades de investigación y evaluación, con el fin de mejorar la salud de las personas y de sus comunidades". En general, todas estas definiciones hacen referencia a las técnicas y tecnologías utilizadas para la práctica médica a distancia, manteniendo el significado etimológico del término telemedicina ("medicina a distancia").

Por otra parte, se define telemedicina clínica como "la obtención de información acerca del estado de salud de un paciente mediante la tecnología de telecomunicaciones y computadores, entre proveedores separados geográficamente o entre proveedores y pacientes, con el objeto de evaluación, diagnóstico, tratamiento o educación"². 
Sin embargo, no debemos considerar la telemedicina únicamente como mera trasmisión de información y comunicación sanitaria entre pacientes y médicos separados en el espacio. Es, además, un medio de comunicación, formación y consulta entre profesionales de la salud, tanto del ámbito hospitalario como de atención primaria, y permite una mejor atención integral del paciente y formación continua de los profesionales de salud.

Se puede considerar que la telemedicina se sirve de tres dimensiones: telecomunicaciones, ciencias de informática y servicios de salud. De esta forma es posible la recolección, procesamiento, transmisión, análisis, almacenamiento y visualización de los datos médicos mas relevantes en un corto periodo de tiempo, disminuyendo el tiempo de espera del diagnostico.

Este suministro de servicios médicos hasta lugares distantes es posible gracias al uso de la tecnología en telecomunicaciones. Se utiliza desde servicios telefónicos estándar (sistemas clásicos) a modelos más evolucionados, y bandas anchas de transmisión de señales digitales conectadas a ordenadores, fibras ópticas, utilización de satélites y otra gran variedad de dispositivos periféricos y software sofisticados. Por tanto, las aplicaciones de telemedicina están evolucionando desde los sistemas clásicos de conexión punto a punto para aplicaciones dedicadas hacia sistemas interactivos de multimedia en red distribuido.

Podemos distinguir dentro de la telemedicina dos modos de operación básicos: a) En tiempo real o modo sincrónico, b) En tiempo diferido o modo asincrónico, o, como también se conoce, el modo de almacenar y enviar. Para la modalidad sincrónica se requiere tener establecidas agendas conjuntas y disponibilidad simultánea de los agentes que estén interviniendo en la sesión. Se utiliza el modo asincrónico en aquellos casos en los que el diagnóstico o la consulta de la información enviada no implican una situación de emergencia, y se puede diferir su consulta en minutos y horas, utilizando por ejemplo el correo como medio de transmisión de la información. Esta modalidad es la que mayor volumen de actividad constituye en la actualidad ${ }^{3}$.

\section{ACTUALIDAD DE LA TELEMEDICINA}

Desde hace años, los sistemas sanitarios buscan nuevas estrategias de organización y alternativas a los modos tradicionales de proporcionar servicios sanitarios. La aparición de las tecnologías de la in- formación y las comunicaciones ha facilitado la viabilidad de modelos organizativos, como la continuidad asistencial o el acercamiento de la atención al entorno del paciente. Posteriormente, la evolución de estas nuevas tecnologías ha permitido aplicar los conceptos de globalidad e interoperabilidad a las organizaciones sanitarias, dando lugar a nuevos entornos organizativos y de trabajo en los que aquel primer concepto de telemedicina asociado, exclusivamente, a la superación de barreras geográficas ha perdido vigencia.

La aparición de nuevos modelos de atención sanitaria con procedimientos novedosos implica la colaboración entre los agentes sanitarios y los propios pacientes, usando medios electrónicos que facilitan a su vez el necesario control de la calidad asistencial, la mejora de la continuidad, de la adherencia a los tratamientos, evitación de eventos adversos y la cuantificación económica del coste sanitario ${ }^{4-5}$.

Aunque se ha descrito el uso de tecnologías de telecomunicación para la provisión de servicios médicos desde los años 60 , es en las dos últimas décadas (desde los años 90) cuando aparece un verdadero interés por la necesidad de encontrar procedimientos alternativos de atención médica que respondan al incremento observado tanto en los costes sanitarios como en las expectativas de los pacientes. Este reciente impulso de la telemedicina ha colaborado en el incremento de los beneficios para el profesional de salud y para los usuarios de los servicios sanitarios.

Son muchas las áreas sanitarias que se han visto influidas en los últimos años por estas nuevas tecnologías, entre otras las consultas de los pacientes a profesionales sanitarios, la comunicación entre profesionales y el seguimiento de pacientes con problemas de salud de carácter crónico.

La asistencia prestada a través de telemedicina brinda la posibilidad de un mejor seguimiento de las enfermedades crónicas y facilita la educación sanitaria del paciente en medicina preventiva y salud publica ${ }^{3}$. Su utilización facilita la accesibilidad de los pacientes a los servicios sanitarios, sin necesidad de tener que realizar desplazamientos, desde su propio domicilio. El actual desarrollo de las TICs (Tecnología de la Información y la Comunicación) permite la obtención de una amplia información de una manera rápida y sencilla ${ }^{3}$.

En la primera encuesta sobre patrones de uso e interés por las nuevas tecnologías de los pacien- 
tes atendidos en unidades de hipertensión arterial (HTA) de toda España ${ }^{6}$, los pacientes de edad media (20-50 años) muestran un elevado porcentaje de utilización de Internet (46-74\%) y un interés por conocer mejor su enfermedad. Los resultados muestran que los pacientes de edad media consideran útiles las nuevas herramientas de la telemedicina para un mejor conocimiento, seguimiento y control de su enfermedad.

Es conocido que se están desarrollando diferentes estudios en los últimos años con el objetivo de evaluar la relación coste-efectividad de la utilización de la telemedicina. Así, en Estados Unidos, los resultados preliminares de algunos programas de salud sugieren la viabilidad y la buena relación coste-efectividad de su utilización en la asistencia sanitaria domiciliaria ${ }^{7}$. Sin embargo, la evidencia disponible hasta hace algún tiempo en nuestro país no permitía confirmar esa relación coste-efectividad favorable ${ }^{8}$.

En la actualidad nos podemos plantear la posibilidad de una utilización más provechosa de todo el potencial que ofrece la nueva generación de sistemas de telemedicina personal y móvil en diferentes ámbitos sanitarios. La disponibilidad de soportes adecuados facilitará el desarrollo mantenido de la Atención Primaria. La implantación de las nuevas tecnologías de telemedicina en Atención Primaria podría formar parte de la estrategia de mejora del modelo actual de prestación de servicios sanitarios a una población que ha cambiado sus características sociodemográficas en los últimos años.

El Centro de Prácticas basadas en la Evidencia de la Universidad de Portland (Oregón) publicó en $2001^{8}$ una revisión para evaluar la eficacia de intervenciones por telemedicina sobre los resultados de salud, comparando sus aplicaciones en el hogar y en la consulta o el hospital. Se mostró evidencia de la eficacia en los resultados clínicos con las aplicaciones domiciliarias para el manejo de enfermedades crónicas, HTA y SIDA. En las aplicaciones hospitalarias encontraron que la telemedicina era comparable a la atención cara a cara en medicina de emergencias, resultando beneficiosa en las unidades de cirugía y cuidados intensivos neonatales, así como en la transferencia de pacientes neuroquirúrgicos.

La posible falta de aceptación de los usuarios de los servicios de la telemedicina puede ser una importante barrera para el despliegue, subrayando la necesidad de que intervengan los usuarios y pro- fesionales de la medicina. Se ha desarrollado una guía de teletratamiento en la que las funciones de médicos, técnicos y usuarios se encuentran, y se han incorporado los principios de la medicina basada en la evidencia, permitiendo la implicación de las diferentes partes interesadas en el diseño de la misma $^{9}$. Esta estrategia debería aumentar la probabilidad de aceptación del usuario.

En el Libro Blanco de la Comisión de las Comunidades Europeas "Juntos por la salud", un planteamiento estratégico para la UE (2008-2013), se plantean tres objetivos que deberían impulsar una nueva estrategia en los aspectos relacionados con la salud: 1) Promover la buena salud en una Europa que envejece, 2) Proteger a los ciudadanos frente a las amenazas a la salud y 3) Fomentar sistemas sanitarios dinámicos y nuevas tecnologías. El texto literal de este tercer objetivo es: "Las nuevas tecnologías pueden revolucionar los sistemas de salud y de atención sanitaria y contribuir a su sostenibilidad futura. La sanidad electrónica, la genómica y las biotecnologías pueden mejorar la prevención de enfermedades y la prestación de tratamiento y favorecer un cambio de la asistencia hospitalaria hacia la prevención y la atención primaria". Se afirma que las nuevas tecnologías pueden mejorar la prevención de las enfermedades y facilitar la seguridad de los pacientes. Es preciso, por tanto, establecer un marco comunitario de servicios asistenciales seguros y de calidad, y establecer medidas para apoyar a los estados miembros en su gestión de la innovación de los sistemas de salud.

\section{VENTAJAS Y DESVENTAJAS DE LA TELEMEDI- CINA}

Un informe sobre evaluación de tecnologías sanitarias del Ministerio de Sanidad muestra las posibles ventajas y desventajas de esta aplicación tecnológica ${ }^{10}$.

\section{Potenciales ventajas}

1. Facilita la equidad en el acceso a los servicios sanitarios, proporcionando una atención médica universal de alta calidad, independientemente de la localización geográfica.

2. Dota a los pacientes de atención médica especializada en aquellos lugares donde no disponen de ella, reduciendo la necesidad de realizar desplazamiento por parte de los pacientes y/o de los profesionales de salud. 
3. Reducción de los tiempos de espera. Menor tiempo en la realización del diagnostico $y$, consecuentemente, menor tiempo en el tratamiento, evitando demoras en los casos graves que pudieran acarrear serios problemas para el paciente.

4. Facilita el manejo precoz de paciente, previo a la llegada de los equipos de emergencia o traslados en ambulancia convencional.

5. La posibilidad de realizar consultas remotas desde atención primaria al hospital, permitiendo a los profesionales mantener un contacto continuo entre ellos, mejorando así su formación y competencias.

6. Reducción de pacientes derivados a consultas especializadas, ofreciendo respuesta a sus problemas de salud desde las consultas de atención primaria, sin necesidad de demoras en tiempo y desplazamientos.

7. Reducción de gastos, evitando al profesional y al paciente la necesidad de realizar desplazamientos.

\section{Ventajas en función del beneficiario}

1. Ventajas para los pacientes:

- Diagnósticos y tratamientos más rápidos.

- Reducción del número de exámenes complementarios.

- Atención integral desde el primer momento.

- Evita la incomodidad de desplazamientos para pacientes y familiares.

2. Ventajas para los médicos de atención primaria:

- Nuevas posibilidades de efectuar consultas con especialistas.

- Posibilidad de evitar inconvenientes desplazamientos.

- Más elementos de juicio a la hora de adoptar decisiones.

- Mejora la calidad de las imágenes para poder diagnosticar.

- Mejora de los circuitos de trasmisión de información, evitando la pérdida de informes.

3. Ventajas para los hospitales:

- Reducción del peligro de pérdida de imágenes.

- Diagnósticos y tratamientos más rápidos y precisos.

- Mejor y más rápida comunicación entre distintos servicios.

- Eliminar el duplicado de información.
- Más eficacia de los equipos y servicios.

- Mayor economía en los gastos derivados del trasportes.

4. Ventajas para el sistema sanitario

- Mejor utilización y aprovechamiento de los cursos.

- Análisis científicos y estadísticos más fáciles.

- Mejor gestión de la salud pública.

- Recursos adicionales para la enseñanza.

\section{Potenciales inconvenientes de la telemedicina}

1. Menor exactitud de ciertas imágenes trasmitidas con telemedicina en relación con las imágenes originales, si las TICs nos son las adecuadas.

2. Aspectos ligados a la seguridad y la confidencialidad en la relación médico-paciente mediante interfaces.

3. Aumento de la demanda a los especialistas, pudiéndose llegar a no poder satisfacer el elevado volumen asistencial de pacientes.

4. Los programas utilizados en telemedicina deben compararse con otras opciones alternativas, asegurándose que además de ofrecer servicios de gran rapidez son a su vez servicios viables.

5. Riesgo de pérdida de datos e imágenes debido a la compresión de dichos datos para aumentar la velocidad de transmisión.

6. La tecnología e infraestructura deben estar lo suficientemente desarrolladas para soportar la implantación de la telemedicina a gran escala.

7. La implementación de los sistemas de telemedicina depende de una adecuada infraestructura de telecomunicaciones.

\section{EXPERIENCIAS Y ESTUDIOS EN TELEMEDICI- NA}

Desde un punto de vista general, los servicios de telemedicina incluyen aplicaciones asistenciales (teleconsulta, telediagnóstico, telemonitorización), las relacionadas con la administración y gestión de pacientes (continuada asistencial, Integración de niveles asistenciales) y las de información y formación a distancia para usuarios y profesionales.

Algunos ejemplos de aplicaciones clínicas ensayadas con éxito incluyen teleradiología, telecardiología, teledermatología o telepsiquiatría. Se emplean servicios de telemedicina en diversos sistemas 
sanitarios y en una variedad de escenarios, tales como zonas rurales, áreas urbanas, áreas sanitarias, prisiones, cuidados a domicilio, emergencias, conflictos bélicos, etc.

Entre las aplicaciones de la telemedicina podemos destacar su empleo en el manejo y seguimiento de algunos factores de riesgo cardiovascular. Se ha usado en el control de la presión arterial en el propio domicilio del paciente, evitando que los pacientes realicen desplazamientos a los centros sanitarios e interferir en su rutina diaria. La automedición de la presión arterial en el domicilio ha demostrado que mejora el cumplimiento terapéutico, el control de la P.A y la adherencia al tratamiento ${ }^{11-12}$. También se ha demostrado la utilidad de la telemedicina en la detección de la presencia de hipertensión arterial ${ }^{13}$. Por otra parte, se ha comprobado el alto grado de satisfacción de los hipertensos con el seguimiento de su patología ${ }^{12}$.

En otro estudio desarrollado en Extremadura, en el que participaron servicios de Atención Hospitalaria (medicina interna y urgencias) y de Atención Primaria, se realizó un seguimiento de diferentes factores de riesgo cardiovascular, incluida la hipertensión arterial y la diabetes. Los participantes fueron seguidos durante 5 meses, comprobando que la utilización de la telemedicina permitió la resolución del $50 \%$ de las llamadas sin precisar derivación a consulta. Los resultados observados permiten a los autores manifestar su apoyo al empleo de esta tecnología en el seguimiento de sujetos que presentan factores de riesgo cardiovascular, afirmando que se trata de una modalidad de asistencia que mejora la relación y la proximidad entre profesionales y pacientes ${ }^{14}$.

Mediante una revisión sistemática se realizó el análisis de los estudios publicados sobre la efectividad de la telemedicina para el diagnóstico y tratamiento de enfermedades oftalmológicas (incluyendo la retinopatía $)^{10}$. Los resultados mostraron una elevada sensibilidad y especificidad de la misma para la detección de retinopatía diabética. Los autores concluyen que el empleo de la imagen digital mediante telemedicina es, por lo menos, tan preciso como la oftalmoscopia realizada por un oftalmólogo experimentado para la detección de la retinopatía diabética.

El uso de la telemedicina en el seguimiento de pacientes con cuadros infecciosos tras el alta hospitalaria ha mostrado que sus beneficios potenciales incluyen ahorros de costes significativos y un período de convalecencia más rápido, ya que los pacientes en su domicilio pueden sentirse mucho más cómodos y participar más activamente en su tratamiento que los pacientes en el hospital ${ }^{15}$. Los mismos autores afirman que los diferentes estudios clínicos han demostrado que la telemedicina utilizada tras el alta hospitalaria es segura y rentable, comparado con el tratamiento hospitalario en enfermos crónicos y agudos.

Existen diferentes aplicaciones de la telemedicina en los distintos Servicios de Salud de nuestro país:

- Andalucía. Creación de un entorno virtual de apoyo a emergencias vitales que ofrece, además, la posibilidad de realizar teleconsulta y formación, implicando a Atención Primaria, Hospitalaria y Sociosanitaria. También se implantado en el hospital Virgen Macarena de Sevilla una "Teleunidad de Lesiones Pigmentarias".

- Baleares. En el hospital Son Llâtzer está en funcionamiento un servicio de diagnóstico remoto de lesiones cutáneas.

- Canarias. Proyecto de telemedicina con teleconsultas en psiquiatría, radiología y dermatología.

- Cantabria. Se dispone de servicio de teleradiología entre distintos hospitales y centros de salud de la comunidad autónoma.

- Castilla-la Mancha. Acceso radiológico desde las consultas de Atención Primaria. Uso de videoconferencia. Proyecto sobre visado electrónico de recetas.

- Castilla-León. Aplicación de telecardiografía para Atención Primaria.

- Galicia. Proyectos de programas de telemedicina aplicada a radiología, oftalmología, dermatología, psiquiatría y neumología.

- Madrid. Se presupuestó en el año 2006 la implantación de diferentes proyectos de telemedicina.

- Navarra. En le año 2006 se presentó el proyecto de prevención de retinopatía diabética por teleconsulta. También se utiliza la teleradiología.

- Otras entidades y actividades. La Agencia Española de Tecnologías Sanitarias realiza un estudio sobre telemedicina en el control de diabetes. Actividades formativas organizadas por diferentes universidades nacionales.

A pesar de la clara intención de incorporar las tecnologías de la información y la comunicación al ámbito sanitario, y al evidente aumento de su aplicación, la telemedicina no disfruta de una presencia mayoritaria en la actividad clínica y asistencial. Se desarrollan un número cada vez mayor de proyec- 
tos, pero son pocos los casos en los que se han incorporado las aplicaciones de telemedicina a los procesos asistenciales ${ }^{16}$. Se atribuye esta dificultad en la implementación a la falta de evidencia científica definitiva sobre su utilidad desde el punto de vista médico y económico ${ }^{17}$.

\section{CONCLUSIONES}

- La utilización de la telemedicina es una alternativa a considerar en la atención del paciente cara a cara.

- La telemedicina nos permite prestar servicios de salud, salvando las barreras geográficas, proporcionando atención especializada en lugares remotos.

- Podemos considerar a la telemedicina como una herramienta no sólo asistencial, sino un mecanismo de trasmitir información y formación entre profesionales de atención primaria, especializada y pacientes.

- La aplicación de la telemedicina puede facilitar la participación de los usuarios, mejorar la calidad del sistema, modificar los hábitos de salud y contribuir a la sostenibilidad del Sistema de Salud.

\section{ALGUNAS PUBLICACIONES ON-LINE DE INTE- RÉS}

La magnitud actual y las perspectivas futuras de la telemedicina han generado un interés que se refleja en el amplio número de publicaciones on-line sobre este tema:

- Journal of Medical Internet Research. Incluido en Medline y órgano de la International Healthcare Coalition; en formato "on line" y de acceso libre, abarca aspectos de investigación, información y comunicación en el campo de la utilización de tecnologías Internet para la provisión de servicios de salud (eHealth).

- Canadian Healthcare Technology Magazine. Revista web editada en Canadá, con noticias y artículos sobre tecnologías y sanidad, de carácter mensual.

- Telemedicine Today Magazine. Principal fuente de información y noticias del sector de proveedores en Telemedicina.

- Virtual Medical Worlds. Revista virtual de tele- medicina y nuevas tecnologías en el ámbito sanitario.

- eHealth International. Revista científica en formato web, asociada a BiomedCentral y de acceso libre a los contenidos; indexada en medline y órgano de la International eHealth Association.

- Medical Informatics and Decisión Making. Publicación online asociada a BiomedCentral, de acceso libre, que recoge artículos de investigación, sometidos a evaluación mediante "peer review" y con procedimientos de publicación rápida; en las áreas de gestión de la información, sistemas y tecnologías en sanidad y el estudio del proceso de toma de decisiones.

- HealthcarelTNews. Publicación electrónica que recoge, con actualización permanente, las noticias del sector de las tecnologías de la información para la salud; publicado en colaboración con el HIMSS (Healthcare Information and Management Systems Society).

- The IPTS Report. Boletín web del Institute for Prospective Technological Studies, editado por el Joint Research Center de la Comisión Europea; disponible en castellano.

- Health Care and Informatics Review Online. Publicada bajo el patrocinio de Health Informatics New Zealand y el Ministerio de Salud de Nueva Zelanda; de acceso libre.

- MOH Health e-News. Publicación del Ministerio de Salud de Nueva Zelanda, país puntero en la utilización de tecnologías de la información y las comunicaciones en el sector Salud.

- Revista eSalud. Revista electrónica de acceso libre, aparecida en marzo 2005; es una iniciativa española orientada al mundo hispano-parlante interesado en acelerar la transformación del sistema, contribuir a la trasformación del sistema sanitario en el siglo 21 mediante el uso eficiente de las tecnologías de la información y las comunicaciones en los Sistemas de Salud.

- Informática y Salud. Revista de la Sociedad Española de Informática de la Salud. Versión WEB de la revista de la SEIS.

- Telehealth Magazine. Dedicada al sector del tratamiento digital de la imagen diagnóstica.

- The British Journal of Healthcare Computing \& Information Management. Versión web de la revista de informática médica británica; ofrece, sin coste, editoriales y resúmenes de algunos artículos.

- Telemedicina Mundial: La salud en el Siglo XXI. Sitio web en español, con artículos, noticias y contenidos sobre actividades y el negocio de la Telemedicina. 
- Military Medical Technology online. Revista de actualidad sobre el uso de las tecnologías en la sanidad militar americana

- Telemedicine, ESA Telecom. Noticias y temas relacionados con Telemedicina en la Agencia Europea del Espacio.

- Journal of Telemedicine and Telecare. Trabajos originales y de investigación sometidos a "peer review". Versión "on line" ofrecida bajo suscripción ó compra de artículos aislados. Sin coste los sumarios y algunos abstracts.

- Telemedicine Journal and e-Health. Trabajos originales y de investigación sometidos a "peer review"; órgano oficial de la American Telemedicine Association. Versión "on line" de acceso mediante suscripción

\section{BIBLIOGRAFÍA}

1. Balas EA, Jaffery FM, Kuperman GJ, Boren SA, Brown $\mathrm{GD}$, Pinciroli FL, et al. Electronic communication with patients: Evaluation of distance medicine technology. JAMA. 1997; 278:152-9.

2. Krupinski E, Nypaver M, Poropatich R, Ellis D, Safwat R, Sapci H. Clinical Applications in Telemedicine/ Telehealth (State-of-the-Art Telemedicine/Telehealth Symposium: An International Perspective). J Telemed Telecare. 2002; 8(1):13-34.

3. Monteagudo JL, Serrano L, Hernández C. La telemedicina:¿ciencia o ficción? An Sist Sanit Navar. 2005; 28:309-303.

4. Sing D. Transforming Chronic Care: Evidence about improving care for people with long-term conditions. The University of Birmingham; 2005. URL: http://www. download.bham.ac.uk/hsmc/pdf/transforming_chronic care.pdf (Visitado: 20-04-2010).

5. WHO. Innovative care for chronic conditions: Building blocks for action. Geneva: WHOrganization; 2002.

6. Campo C, Naval J, Ruilope LM, Segura J, Figueras M, Sánchez R, et at. Resultados de la primera encuesta sobre patrones de uso e interés por las nuevas tecnologías de los pacientes atendidos en unidades de hipertensión en España. Hipertensión. 2003; 20:50-5.
7. Kaye LW. Telemedicine: extensión to home care. Telemed J. 1997; 3:243-6

8. Hersh WR, Helfand M, Wallace J, Kraemer D,Patterson $P$, Shapiro S, et al. Clinical outcomes resulting from telemedicine interventions: a systematic review. BMC Medical Informatics and Decision Making. 2001; 1(1):5.

9. Huis in't Veld RM, Widya IA, Bults RG, Sandsjö L, Hermens HJ, Vollenbroek-Hutten MM. A scenario guideline for designing new teletreatments: a multidisciplinary approach.J Telemed Telecare. 2010; 16(6):302-7.

10. Orruño E, Lapuente JL, Gutiérrez A, Asua J. Análisis de la introducción de la Telemedicina en la gestióncoordinación de atención primaria-especializada. Evaluación de resultados y costes de experiencias preexistentes (teleoftalmología). Madrid: Plan Nacional para el SNS del MSC. Servicio de Evaluación de Tecnologías Sanitarias del País Vasco; 2006. Informes de Evaluación de Tecnologías Sanitarias: OSTEBA № 2006/07.

11. Bayo J, Rosa C, Dalfó A, Naberan K. Automedida de la presión arterial domiciliaria y telemedicina. ¿Qué nos depara el futuro? Aten Primaria. 2005; 35:43-50.

12. Friedman RH, Kazis LE, Jette A, Smith MB, Stollerman $\mathrm{J}$, Torgerson J, et al. A telecommunications system for monitoring and counseling patients with hypertension. Impact on medication adherence and blood pressure control. Am J Hypertens. 1996; 9:285-92.

13. Rogers MA, Small D, Buchan DA, Butch CA, Stewart $\mathrm{CM}$, Krenzer BE, et al. Home monitoring service improves mean arterial pressure in patients with essential hypertension: a randomized, controlled trial. Ann Intern Med. 2001; 134:1024-32.

14. Romero JM, Calvo JM, Arévalo JC, Pérez JN, Ortiz $\mathrm{C}$, Gutierrez $\mathrm{C}$ et al. Control de los factores de riesgo cardiovascular mediante telemedicina. Hipertensión. 2005; 22:89-90

15. Eron L. Telemedicine: the future of outpatient therapy? Clin Infect Dis. 2010; 51 Suppl 2:224-30.

16. Broens TH,Huis RM, Vollenbroek-Hutten MM, Hermens HJ, van Halteren AT, Nieuwenhuis LJ. Determinants of successful telemedicine implementations: a literature study. J Telemed Telecare. 2007; 13:303-9.

17. Grigsby J, Brega AG,Devore PA. The evaluation of telemedicine and health services research. Telemed $J$ E Health. 2005; 11:317-28. 